\title{
How to Support Newcomers Onboarding to Open Source Software Projects
}

\author{
Igor Steinmacher $^{1}$ and Marco Aurélio Gerosa ${ }^{2}$ \\ ${ }^{1}$ DACOM - UTFPR Campo Mourao - PR - Brazil \\ igorfs@utfpr.edu.br \\ ${ }^{2}$ IME - USP Sao Paulo - SP - Brazil \\ gerosa@ime.usp.br
}

\begin{abstract}
While onboarding an open source software (OSS) project, contributors face many different barriers that hinder their contribution, leading in many cases to dropout. Many projects leverage the contribution of outsiders and the sustainability of the project relies on retaining some of these newcomers. In this research, we aim at understanding the barriers that hinder onboarding of newcomers to OSS projects, by means of different empirical approaches, and proposing a set of strategies that can be used to support the first step of newcomers.
\end{abstract}

\section{Introduction}

Open Source Software (OSS) communities are generally self-organized and dynamic, counting with contributions of volunteers spread all over the globe. These communities demand a high influx of newcomers to keep alive [1]. According to Qureshi and Fang [2], motivate, engage and retain developers is a way to foster a sustainable amount of developers in a project. However, newcomers face difficulties and obstacles when they start interacting to the project, resulting in a high dropout rate [5]. Newcomers need to learn both social and technical aspects of the project, exploring mailing lists, wikis, issue trackers and source code repositories [3].

In a previous study [6], some developers who tried to onboard in two well-known OSS projects reported the obstacles they faced. Developers indicated that the lack of awareness and guidance during the course of their first steps (setup and choosing the right mean to start) discouraged further contributions. Therefore, a major challenge for OSS projects is to provide ways to support the onboarding of newcomers.

The goal of this research is to explore the barriers faced by newcomers to onboard to OSS projects and analyzing which strategies can be used to support newcomers to overcome these barriers. The overall question to be answer in this thesis is: "How to support the onboarding of newcomers in OSS projects?" To guide answering this question, some specific research questions were defined for this thesis:

- What are the barriers faced by newcomers when onboarding OSS projects?

- What are the forces that influence the joining and retention of developers in OSS projects?

- What strategies can be used to help newcomers?

L. Corral et al. (Eds.): OSS 2014, IFIP AICT 427, pp. 199-201, 2014.

(C) IFIP International Federation for Information Processing 2014 


\section{Research Design}

The research of this thesis uses different empirical methods to answer the research questions defined. This research is a result of the combination of systematic literature reviews, interviews, case study and experiments. The research design is composed of three phases and some studies, as presented in Figure 1, described below:

- Warm Up. This phase consists of one case study (S1) conducted in order to motivate the problem addressed by this thesis and to investigate whether absence of response, politeness, usefulness or the author of answers influence the onboarding of newcomers in an OSS project.

- Phase I - Find Barriers. This phase is composed of three studies that objective to raise what are the barriers that hinder newcomers onboarding to OSS projects. We will empirically gather the barriers from the literature (S2), by means of Systematic Literature Review, and from the OSS community (S3), using interviews with project members and feedbacks from newcomers. The results obtained in the systematic review and in the qualitative analysis will be confirmed by means of a survey to be administered with OSS community members (S4).

- Phase II - Proposing Support to Newcomer Onboarding. In this phase, the goal is to consolidate the barriers that hinder newcomers onboarding to OSS and map them to proposed strategies that can support newcomers overcoming them. We plan to propose solutions based on: (i) suggestions and studies that emerged during Phase I; (ii) systematic review on awareness mechanisms (S5); and (iii) state-of-the-practice, by analyzing the strategies that some projects currently adopt to support newcomers.

Based on the results of the survey conducted during Phase I, we will select a subset with the most relevant barriers to further explore in this phase. We plan to design and conduct a controlled experiment to assess the effectiveness of using the proposed solutions to the selected barriers (S6).

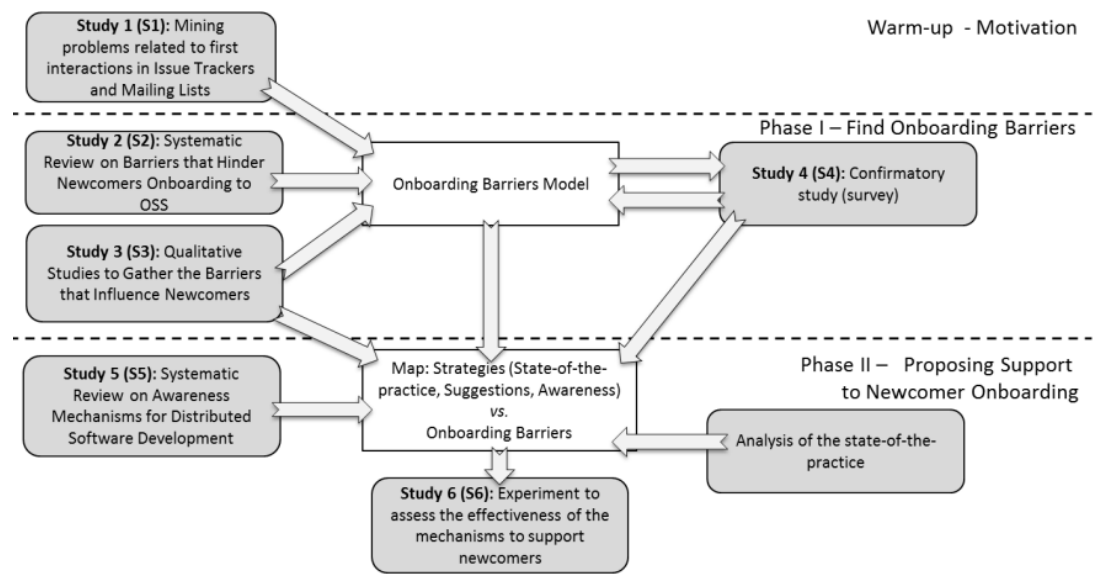

Fig. 1. Research Design 


\section{Preliminary Results}

In order to situate our problem and delimit the scope of this thesis, we analyzed the existent literature and proposed a model [4] that represents the stages (onboarding and contributing) that are common to and the forces that are influential to newcomers being drawn or pushed away from a project.

We conducted a case study [5] to analyze newcomers dropout reasons. We collected five years of communication of the list of emails from developers and discussions from the task manager (Jira). There was no indication that the number of responses influences the withdrawal. We found evidence that receiving inadequate answers affects the decision of newcomers to abandon the project

We also collected feedback from some developers that tried to contribute to two OSS projects [6]. The developers reported some demotivating facts: unanswered emails, outdated documentation, outdated issues that resulted in waste of time and flaming threads.

We conducted a systematic literature review aiming at identifying the barriers faced by newcomers to OSS projects. As a result, we provided a hierarchical model that relies on five categories: social interactions, newcomers' previous knowledge, finding a way to start, documentation problems, and code issues. Some barriers that hinder newcomers to OSS were also identified using a qualitative analysis on data obtained from newcomers and members of OSS projects. The results enabled us to create a model composed of 38 problems, grouped into seven different categories.

Acknowledgements. The authors thank CAPES (BEX 2038-13-7) and CNPq (process 477831/2013-3) for the financial support. Marco Gerosa receives a grant from the CNPq and FAPESP.

\section{References}

[1] Park, Y., Jensen, C.: Beyond pretty pictures: Examining the benefits of code visualization for Open Source newcomers. In: 5th Intl. Workshop on Visualizing Software for Understanding and Analysis, pp. 3-10 (2009)

[2] Qureshi, I., Fang, Y.: Socialization in Open Source Software Projects: A Growth Mixture Modeling Approach. Org. Res. Methods. 14(1), 208-238 (2011)

[3] Scacchi, W.: Understanding the requirements for developing open source software systems. Software, IEE Proceedings 149(1), 24-39 (2002)

[4] Steinmacher, I., Gerosa, M.A., Redmiles, D.: Attracting, Onboarding, and Retaining Newcomer Developers in Open Source Software Projects. In: Workshop on Global Software Development in a CSCW Perspective (2014)

[5] Steinmacher, I., Wiese, I., Chaves, A.P., Gerosa, M.A.: Why do newcomers abandon open source software projects? In: Intl. Workshop on Cooperative and Human Aspects of Software Engineering (CHASE), pp. 25-32 (2013)

[6] Steinmacher, I., Wiese, I.S., Gerosa, M.A.: In: 2012 Third Intl. Workshop on Recommendation Systems for Software Engineering (RSSE), pp. 63-67 (2012) 